\title{
EXPLORING THE CONTEXT AND PRACTICES OF EXPERT SIMULATION MODELLERS
}

\author{
Ahmed, R. ${ }^{*} \&$ Shah, M.* \\ *Lahore School of Economics, Burki Road, Lahore, Pakistan \\ ${ }^{* *}$ Lancashire Business School, University of Central Lancashire, UK \\ E-Mail: rizwan.ahmed@lahoreschool.edu.pk,mhshah@uclan.ac.uk
}

\begin{abstract}
Simulation modelling lacks a rich body of literature on practices of modellers in the real world. We study the context and some generic practices of expert simulation modellers to discover how the context of modellers may affect the practice of modelling and simulation. The results highlight that simulation modellers develop their models under a variety of contexts and their practices may be affected by their context. The problem area, the scope and the breadth of a problem, simulation software and the size and complexity of the model are some of the contextual factors which may affect a modeller's practices such as model development, documentation, maintenance and evaluation. For example, model maintenance is required only for large scale models developed for long term use. Similarly, varying level of documentation may be required depending on the client requirements and project needs. Our study is a valuable addition to the research investigating simulation practice in the real world.
\end{abstract}

(Received in May 2014, accepted in November 2014. This paper was with the authors 2 months for 2 revisions.)

Key Words: Simulation Practice, Simulation Context, Contextual Factors, Modelling Process

\section{INTRODUCTION}

Modelling and simulation, a widely used tool, is considered to be the technical heart of the operational research and management science [1]. However, simulation modelling research generally focuses more on the application of simulation and less on the practice and methodology of modellers in the real world. Since modellers approach modelling differently under varying contexts the outcome of simulation may depend very much on the personal style and practices of a simulation modeller [2]. Robinson [3] suggests that the way a modeller develops his model depends on the characteristics of a model (e.g. size, complexity, objectives, scope, model life, simulation technique, simulation software, team size, etc.) and a modeller's skills (e.g. experience, education, style, team skills and size). Thus, the modelling practices (modelling process, documentation, maintenance, client contact, etc.) may also vary with the characteristics of models and the modeller's skills. Other researchers such as [4-7] suggest that practices of a simulation modeller can potentially affect the quality and success of simulation studies. Simulation practice can only be improved if we understand a modeller's practices in the real world. Therefore, it is important to conduct in-depth studies in order to enhance our understanding of simulation modelling in varying contexts; which in turn may help in improving simulation practice. This paper explores the context and practice of expert simulation modellers and relates how simulation practice may be affected by the context. This research provides a valuable contribution towards enhancing our understanding of simulation practice in the real world.

The paper has been organised in 7 sections. Section 2 provides an overview of the background literature, section 3 outlines the research methodology, sections 4 and 5 present the results of the study, section 6 provides a discussion on the results and Section 7 concludes the paper. 


\section{LITERATURE BACKGROUND}

Despite an abundance of literature on application of simulation in various areas of business and engineering, in-depth studies on simulation practice in the real world are rare and difficult to pursue. A number of surveys exploring modellers' characteristics $[8,9]$ and practice $[10$, 11] have been reported in simulation literature. Arguably, these quantitative studies have provided useful indicators to understand characteristics of modellers and their backgrounds; nevertheless, these studies do not provide deep insights into their practices. We have, however, found a few in-depth studies looking at the practices of modellers under an experimental setting which includes Tako and Robinson [12], Willemain [13], Wang and Brooks [14], and Foss et al. [15]. Each study is described briefly below.

Tako and Robinson [12] highlight the differences in modelling practices of discrete-event simulation (DES) and systems-dynamics (SD) modellers. They conclude that SD modellers spend more time on conceptual modelling as compared to DES modellers, whereas DES modellers spend more time on coding and validation of simulation model as compared to SD modellers. They also suggest that DE modellers seem to follow a more linear thinking approach as compared to an SD modeller; however, both DES and SD modellers follow a highly iterative process of modelling. Furthermore, they note that DES and SD modellers also differ in thinking with regards to model objectives, model complexity and size, inputs data and experimentation. This suggests that potential differences in contextual factors lead to differing modelling practices.

The most prominent in-depth study of modelling practice has been conducted by Willemain $[3,13]$ which explores the way expert modellers develop mathematical models. They analysed expert modellers' practice in five generic categories: context (problem formulation), structure (or design), realization (deriving solution), assessment (testing/validation), and implementation. Willemain [13] identified that experts moved between structuring (design) and assessing (validation) in an iterative fashion and switched their attention between different topics quite frequently. Willemain [13] highlighted that it is important to further improve our understanding on how expert modellers develop their models and suggested that practical guidelines for model formulation should be developed for novices in order to become experts. Willemain [13] acknowledged the limitation of the study as being a one hour experiment and emphasised that future studies should look at modellers' practice in real projects.

More recently, Wang and Brooks [14] conducted a similar study which covered complete real time simulation projects for a client. One expert and nine novices participated in their study. They studied one expert over a period of 10 weeks who worked on a call centre simulation project. The expert recorded the amount of time spent on each category identified as identified by Willemain [13]. A similar set of experiments was conducted on 9 students (as novice modellers) who worked on a real time simulation project for 12 weeks. Each participant worked on a different project. They concluded from their experiment that their expert modeller spent a higher proportion of time on conceptual modelling and validation and verification; whereas novices spent more time on data collection. However, it is important to note that the lack of uniformity in the analysis is an inherent limitation in carrying out such research on a real time project, because each problem in a real time project is different and each modeller may have different personal style and characteristics. This implies that contextual factors have an effect on the way modellers go about developing their models. Wang and Brooks [14] suggest that in a laboratory setting, however, the researcher can perform analysis more uniformly as Willemain [13] did.

Foss et al. [15] interviewed 16 expert modellers from Germany and Norway working in process industries. In this study, instead of giving them a standard case study, the participants 
were asked to discuss how they had developed a model for a particular problem in the past. They interviewed the experts on a pre-defined task structure which included initial data collection, tools selection, conceptual modelling, model representation, implementation, verification, initialization, validation, documentation and model application. Foss et al. conclude that the degree of sophistication of a modelling tool greatly affects the efficiency of the modellers and the quality of the outcome; they also report that modelling seems to be an individual creative activity, heavily dependent on personal style, background, and characteristics of the modeller. They also note that the more experience a modeller has, the less inclined (s) he will be to develop a conceptual model. They also identify that a conceptual modelling style also depends on a modeller's background e.g. control engineers prefer block diagramming, mathematicians think in equations and chemical engineers think in engineering concepts. They say that most simulation models developed by their participants are poorly documented and therefore, rarely reused. They further state that poor documentation makes it very hard to maintain the models.

As the above discussion suggests, a modeller may develop a number of simulation projects of varying complexity, size, and application areas; using different types of simulation software tools and simulation paradigms while dealing with a variety of clients with varying objectives and goals. Therefore, their modelling practice may differ depending on these contextual factors. Our study is a valuable addition in the research investigating simulation practice in the real world. Our study, however, differs from the above mentioned studies in two important ways.

- Firstly, Willemain [13] and Wang and Brooks' [14] experiments explore the practices of simulation modellers but do not pay much attention to the contextual factor; while our particular focus is to investigate how contextual factors of modellers relate with their simulation practice.

- Secondly, the above mentioned studies either use an experimental setting or explore modelling practice under certain pre-defined categories; while we aim to discover modellers practice under their typical context without enforcing an experimental setting and a standardized case study.

We believe that investigating the contexts and practice simulation modellers will enable further understanding of simulation practice and underpin the simulation methodology research.

\section{METHODOLOGY}

We conducted semi-structured interviews with the participants. The semi-structured interviewing technique was the most appropriate for this research as we wanted to explore the contexts and practices of simulation modellers in-depth and generally in a structured manner. The interview questions focused on 8 main areas of interest to explore the modelling context and practices of the modellers.

A pool of interview questions was prepared, consisting of some main open ended questions and several auxiliary questions which were to be asked depending on the flow of interview. A questionnaire consisting of open ended questions was sent to the participants a week prior to conducting the interviews. We also prepared an interview script document, which was used during the interview to ensure a generally uniform way of conducting interviews with all the participants.

We also conducted an extensive pilot study consisting of two phases with an objective to evaluate the interviewing instrument. The first phase aimed to pre-test the validity of questions to be asked in the interview. The initial draft of the questions pool was then improved on the basis of feedback received by the pre-testing participants. In the second 
phase, pilot interviews were conducted with four other participants, which helped us in assessing the appropriateness, structure and flow of the interview. This also provided us with practice for the main set of interviews along with helping us in determining an appropriate duration of the interviews and evaluating the audio recording equipment.

\section{THE PARTICIPANTS AND THEIR CONTEXT}

The participants in this study consist of both simulation practitioners and researchers. Twenty participants took part from the USA, UK, Germany, Spain and South Africa. Table I provides a summary of participants' background.

Table I: Participants' demographics and context.

\begin{tabular}{|c|c|c|c|c|c|c|c|c|}
\hline $\begin{array}{l}\text { Partic } \\
\text { ipants }\end{array}$ & Education & $\begin{array}{l}\text { Experience } \\
\text { (years) }\end{array}$ & Role & Size & Complexity & Techniques & $\begin{array}{c}\text { Simulation } \\
\text { software }\end{array}$ & $\begin{array}{c}\text { Model's } \\
\text { perceived life }\end{array}$ \\
\hline A1 & Bachelor & 2 & $\mathrm{R}$ & Small & Low & $\mathrm{DE}$ & Extend & Short-term \\
\hline A2 & $\mathrm{PhD}$ & 2 & $\mathrm{R}$ & Medium & Medium & $\mathrm{DE}$ & $\begin{array}{l}\text { GPSS, Extend, } \\
\text { Excel }\end{array}$ & Short-term \\
\hline A3 & $\mathrm{PhD}$ & 3 & $\mathrm{C}$ & Medium & High & DE, SD & Extend & Long-term \\
\hline A4 & $\mathrm{PhD}$ & 9 & $\mathrm{C} / \mathrm{R}$ & Large & High & DE, SD & Extend, Vensim & Both* \\
\hline A5 & $\mathrm{PhD}$ & 9 & $\mathrm{C} / \mathrm{R}$ & Large & Medium & SD & Vensim, Java & Both* \\
\hline A6 & Masters & 4 & $\mathrm{C}$ & Medium & High & DE, SD, HB & Extend & Both* \\
\hline A7 & $\mathrm{PhD}$ & 6 & $\mathrm{R}$ & Small & Low & SD & $\begin{array}{l}\text { SimuLink, Matlab, } \\
\text { Vensim, PowerSim }\end{array}$ & Short-term \\
\hline A8 & $\mathrm{PhD}$ & 16 & $\mathrm{C}$ & Medium & Medium & $\mathrm{DE}, \mathrm{SD}, \mathrm{HB}$ & Extend & Long-term \\
\hline A9 & $\mathrm{PhD}$ & 11 & $\mathrm{C}$ & Medium & Medium & $\mathrm{DE}, \mathrm{SD}$ & Vensim, iThink & Both* \\
\hline A10 & $\mathrm{PhD}$ & 20 & $\mathrm{C} / \mathrm{R}$ & Medium & Medium & $\mathrm{DE}, \mathrm{SD}, \mathrm{SB}$ & Extend & Both* \\
\hline A11 & $\mathrm{PhD}$ & 13 & $\mathrm{C}$ & Medium & Medium & $\mathrm{DE}$ & Witness & Both* \\
\hline A12 & Masters & 2 & $\mathrm{C} / \mathrm{R}$ & Medium & Medium & $\mathrm{DE}$ & $\begin{array}{l}\text { Witness, Visual } \\
\text { Basic }\end{array}$ & Both* \\
\hline $\mathbf{A 1 3}$ & Bachelor & 7 & $\mathrm{C}$ & Medium & Medium & $\mathrm{DE}$ & Witness & Short-term \\
\hline $\mathbf{A 1 4}$ & $\mathrm{PhD}$ & 6 & $\mathrm{C}$ & Medium & Medium & $\mathrm{DE}$ & Witness, C\#, Sim8 & Short-term \\
\hline A15 & $\mathrm{PhD}$ & 14 & $\mathrm{C}$ & Medium & High & $\mathrm{DE}, \mathrm{SD}$ & SimScript & Both* \\
\hline A16 & Bachelor & 8 & $\mathrm{C} / \mathrm{R}$ & Large & High & $\mathrm{DE}, \mathrm{SD}$ & Swaim, Extend & Both* \\
\hline S17 & $\mathrm{PhD}$ & 10 & $\mathrm{R}$ & Medium & Medium & $\mathrm{DE}, \mathrm{SD}$ & Pascal, Simul8 & Short-term \\
\hline A18 & $\mathrm{PhD}$ & 1 & $\mathrm{C} / \mathrm{R}$ & Medium & Medium & $\mathrm{DE}$ & Witness & Both* \\
\hline A19 & $\mathrm{PhD}$ & 9 & $\mathrm{R}$ & Small & Low & SD & QSim & Short-term \\
\hline $\mathbf{A 2 0}$ & Masters & 6 & $\mathrm{C}$ & Medium & Medium & $\overline{\mathrm{DE}}$ & Witness & Short-term \\
\hline \multicolumn{2}{|c|}{$\begin{array}{l}\text { Average } \\
\text { experience }\end{array}$} & \multicolumn{7}{|l|}{8.5 years } \\
\hline \multicolumn{2}{|c|}{$\begin{array}{l}\text { Education } \\
\text { summary }\end{array}$} & \multicolumn{3}{|c|}{$\mathrm{PhD}$ (14), Masters (3), Bachelor (3) } & \multicolumn{2}{|c|}{ Modelling technique } & \multicolumn{2}{|l|}{$\begin{array}{l}\text { DES: (8), SD: (4), } \\
\text { Both DES and SD: (8) }\end{array}$} \\
\hline \multicolumn{2}{|c|}{$\begin{array}{l}\text { Professional role } \\
\text { summary }\end{array}$} & \multicolumn{3}{|c|}{$\begin{array}{l}\text { Consultant (9), Researcher (5), } \\
\text { Researcher/ Consultant (6) }\end{array}$} & \multicolumn{2}{|l|}{ Size } & Small: 3, Medium: 14, & Large: 3 \\
\hline Model & & $\begin{array}{l}\text { Short-term (8), } \\
\text { Long/Short-tert }\end{array}$ & $\begin{array}{l}\text { ong-ter } \\
(10)\end{array}$ & & Complexity & & Low: 3, Medium: 12, $\mathrm{I}$ & High: 5 (25\%) \\
\hline Summe & ry of types of & models & & & & & & \\
\hline Aims: & $\begin{array}{l}\text { ocess insigh } \\
\text { aprovement, }\end{array}$ & $\begin{array}{l}\text {, forecasting } \\
\text { uality assurar }\end{array}$ & roce & $\begin{array}{l}\text { and sche } \\
\text { performa }\end{array}$ & $\begin{array}{l}\mathrm{e} \text {; resource pl } \\
\text { measuremen }\end{array}$ & $\begin{array}{l}\text { nning and all } \\
\text { and monitori }\end{array}$ & $\begin{array}{l}\text { location, process evalu: } \\
\text { ing, and process design }\end{array}$ & ation and \\
\hline Applic & tion area: & $\begin{array}{l}\text { cess improve } \\
\text { management }\end{array}$ & $\begin{array}{l}\text { opti } \\
\text { ucatio }\end{array}$ & $\begin{array}{l}\text { sation, an } \\
\text { and trainir }\end{array}$ & $\begin{array}{l}\text { olanning; tech } \\
\text { control and o }\end{array}$ & $\begin{array}{l}\text { ology evaluat } \\
\text { erational mar }\end{array}$ & $\begin{array}{l}\text { ation and adoption; pro } \\
\text { inagement }\end{array}$ & ject planning \\
\hline Proble & n domain: $\mathrm{se}$ & $\begin{array}{l}\text { ety control sys } \\
\text { tres, manufact } \\
\text { lth care, softw }\end{array}$ & $\begin{array}{l}\text { ns, oil } \\
\text { ing, fin } \\
\text { e devel }\end{array}$ & $\begin{array}{l}\text { ad gas pipe } \\
\text { ncial servic } \\
\text { pment proc }\end{array}$ & $\begin{array}{l}\text { ines, mining, st } \\
\text { es, defence (we } \\
\text { sses, science (1 }\end{array}$ & $\begin{array}{l}\text { pply chain an } \\
\text { pons, vehicle } \\
\text { hysical, bioin }\end{array}$ & $\begin{array}{l}\text { ad logistics, airport pro } \\
\text { es), telecom, retail, roa } \\
\text { aformatics) }\end{array}$ & $\begin{array}{l}\text { cesses, call } \\
\text { d and traffic, }\end{array}$ \\
\hline
\end{tabular}

The participants can be categorized into three groups; researchers (R), consultants (C), and researchers cum consultants $(\mathrm{C} / \mathrm{R})$; each group may have different intents to simulation modelling. For example, researchers' objective for simulation model development is usually innovation. Whereas consultants' intents to simulation model development are commercial. Modellers' objectives in model development may affect the way they develop simulation models. Therefore, the inclusion of researchers and consultants is aimed to discover state-ofthe-art simulation practice based on the practices of both researchers and consultants. 
Table I summarises participants' demographics and shows that there are 14 participants with a $\mathrm{PhD}, 3$ participants with Master degrees and 3 participants who hold a Bachelor degree. This suggests that the participants in this study are highly educated and most of them had some modelling education as part of their professional or research degrees. Table I also shows that 5 participants are simulation researchers, 9 participants are simulation consultants and 6 participants are researchers cum consultants. This indicates that the participants in both groups are a good mix of researchers and consultants. The average experience of the participants in simulation is 8.5 years. This suggests a high level of simulation experience amongst the participants.

These results include the type of simulation models developed by the participants, the modelling tools used by them, the modelling techniques used, their size and the complexity of the models produced. This contextual data provides information about important factors which in turn affects the practices of the simulation modellers and their modelling process. Table I summarises the contexts of the participants; detailed discussion is provided in subsequent sections.

\subsection{Types of models}

Our participants develop their simulation models with a variety of aims; in various application areas and problems domain. As Table I shows, insights into and understanding the business processes, cost, schedule, quality forecasting, resource allocation \& planning and performance monitoring \& evaluation are the main aims of the models developed by our participants. Our participants develop simulation models both for services and manufacturing; which include transportation and passenger flow control, supply chain and logistics management, oil and gas pipelines, mining, call centre management, telecom, banking and financial services, healthcare policy planning, defence and software development processes.

The life of the simulation models developed by the participants includes both short term and long term models. Short term models are those which are used only for days, weeks or months and long term models are used for years. Table I shows that only 2 participants develop models for long term use, whereas 8 participants develop models which are used for short term: the majority, 10 participants, develop models both for short and long term use.

\subsection{Simulation software}

A number of different simulation software and programming languages are used by our participants to develop their simulation as shown in Table I. Simulation software used are Extend, Witness, Arena, Simul8, GPSS, Swaim, MS Excel, SimuLink, iThink, Vensim, SimScript, Matlab, QSim and ModSim; and the programming languages used are Visual Basic, Java and Pascal. Witness, Extend and Vensim are the most popular simulation software amongst these participants. Table I show that discrete event (DE) and system dynamics (SD) techniques are used by the majority of the participants. Out of 20 participants, 3 participants use SD, 8 participants use DE, and 9 participants use both DE \& SD; providing a good mix of modellers using both techniques.

The participants discussed some merits and demerits of the simulation software they use. Our participants also believe that the right choice of simulation software for a given problem is very important for successful simulation projects. For example, A16 believes that a wrong choice of simulation software may introduce a lot of unnecessary complexity in a simulation model. A6 shares his personal experience of choosing wrong simulation software which resulted in late delivery and cost overrun. The selection of a tool may depend on different factors such as; budget constraints, performance requirements of the simulation model, ease of using the tool, maintenance support, documentation support and design quality, as suggested by the participants. 


\subsection{Size and complexity of simulation models}

The participants of this study developed simulation models of varying complexity and size. The metrics for size and complexity of the models is very subjective. The participants indicate various measures to assess a simulation model's size and complexity; however, there seems to be no agreement as to what can be a realistic measure for size and complexity. Table I summarises participants' perceptions about the size of models they develop.

Table I shows that 3 participants think that they develop small models; 14 participants develop medium sized models; and 3 participants develop large models. This suggests that most of the participants in this study perceive that they develop simulation models of small and/or medium size.

Most participants like to perceive the size of the model in terms of magnitude of the problem i.e. number of entities, elements, activities or process steps; where entities and elements mean a conceptual or physical part of the system under study, such as machines, belts, people, or process steps etc. Some participants like to measure model size in terms of the time it takes to develop the model as shown by the transcript excerpt of A12 in Exhibit 1. Participants who use Witness or Extend also tend to measure size in terms of the number of 'blocks' in the model. Some participants also think that the number of variables can be a measure of size. Few participants think that "lines of code" is a good measure for the size of a simulation model. A12, A14 and S17 think that the amount of input data can also be used to measure size.

According to the participants, the size of a model also depends on the simulation software or the programming language being used to develop the model. A big model developed in one simulation software may appear to be a small model in another. For example, a model developed in Java or Visual Basic may appear to be very big in terms of lines of code; however, when developed in Witness or Extend, it may appear to be smaller due to the availability of a model constructs library and visual components. Therefore, A1 suggests that when estimating size of the model, simulation software should also be taken into consideration.

According to the participants, the size of a model also depends on the simulation software or the programming language being used to develop the model. The results show that there are no agreed metrics for simulation model size and no significant debate can be found about simulation model size in the literature.

Table I shows that 3 participants develop simulation models of low complexity; 12 participants develop models of medium complexity; and 5 participants develop highly complex models. This shows that most of the participants in both groups mostly develop simulation models of low and/or medium complexity.

Most of the participants wanted to talk more about the complexity of the models rather than size, as quotes from A9 and A12 show in Exhibit 2. The number of interactions between model elements, blocks or the variables is the most popular measure perceived by the participants. Those with continuous simulation background think that the higher the number of feedback loops, the higher the complexity of the model would be. A4 thinks that the number of questions to be answered by the simulation study could be a measure of complexity because the greater the number of questions the greater the output values and analysis, hence making the simulation more complex. Some of the participants also believe that complexity in the data and complexity in the output, originating from a simulation problem, are also good indications of the complexity of the simulation model. A1 and S17 assume that the number of flows in a simulation model can also be a measure for complexity.

Most of the participants think that size and complexity are related most of the time: in general, the larger the model size, the higher will be the complexity. Only A6 and A11 think that size and complexity are not necessarily related. A11 said that a model may be very big in 
terms of input data, number of blocks and variables, but different parts of a model may be replicating a similar structure, therefore, the model may not be as complex as it seems.

Exhibit 1: Participants' views about model size.

A6: I guess number of blocks is one way to characterise the size of a model. And it was a couple of hundred blocks, I won't say it was a huge model. But it was relatively complicated by a medium size model. In terms of the number of the blocks in the model.

A8: Well I am consultant I measure in dollars...

A11: I would say if you are talking about elements rather than variables... if you got something like 10 to 15 elements you got a medium size model... less than 10 probably small... more than 20 means big... and what I mean by elements is machines, parts, buffers...

A12: I think it is natural for consultants (to measure in terms of time), because we would tend to when it comes to communicating with the client that how big the project is, and establishing its cost, we deal in the amount of time it takes to develop it... so yes we tend to talk in terms of development time... I think there is a fairly direct correlation between the amount of time it takes and how difficult and how complex it is to develop it...

A14: ... so you are looking at 2 to 3 thousand servers within the simulation....and a workload of 10 s of thousands of calls per day... so in terms or event list it is quite a big simulation problem..

Exhibit 2: Participants' views about complexity.

A9: I think model complexity is probably more interesting (than model size)... but the point is that there is no agreed measure...

A2: I think there is a fairly direct correlation between the amount of time it takes and how difficult and how complex it is to develop it... it (size) isn't something that I find to be terribly important, what I find important is how long it takes..

A6: To me a model... the important thing about model is its degree of complexity... so complexity can be measured by the relationships amongst variables... my model probably had about 100 variables and it probably had 500 interrelationships amongst the variables... so that's where the complexity came in...

The results in this section show that most of the participants develop simulation models of low/medium complexity. Again there are no agreed metrics to measure the complexity of the models, and similarly, no significant debate can be found in the literature about the complexity of models. However, the results show that complexity of models largely depends on the complexity of problems and size of the models.

\section{PRACTICES OF THE PARTICIPANTS}

\subsection{Model development practice}

Our participants typically develop their simulation models alone, while on rare occasions they collaborate with other modellers; however, they seldom work on the same model concurrently. Only A16 discussed his experience of a large scale defence simulation project where about 200 people worked on various parts of the same project. A5, A9, A10, A11, A12 and A13 share their experience of working on some projects where roles and responsibilities were divided amongst many team members as a simulation modeller, data collector, and process-mapper/system-engineer. According to A12, modellers tend to develop their models more efficiently when they work alone, since having multiple people on one model introduces time and communication overheads. A12, A15 and A16 suggest that communication is the biggest problem since everyone needs to have the same level of understanding about the project; and it becomes even a bigger problem if team members come from different educational, lingual and cultural backgrounds.

All participants say that typically, they define simulation goals and scope, identify inputs and outputs, draw a diagram if needed and start with a small model. Only a few of the participants mention simulation model design as part of their process. Only three participants talked about design as a process step; these participants claim to be developing big and highly 
complex models. Most of the participants suggest that an evolutionary approach should be adopted for model development; by starting small and adding details as requirements, the scope of the model becomes clearer and validation and verification should be conducted alongside.

Heavy client contact is emphasized by most of the participants who develop models for a client. Since researchers often do not have a client, unless it is a project in collaboration with industry, the researchers do not emphasize client contact for successful simulation studies. In the commercial world, client wants to see the solution of their problems as quickly as possible; therefore, the participants recommend a rapid approach towards model development. Thus, a simulation modeller must involve the client heavily and adapt his/her modelling process according to the client needs in order to deliver the results and recommendation quickly.

Exhibit 3: Participants' perceptions about teamwork.

\begin{abstract}
A12: It is partly nature of the project and I think it is part of nature of modelling (also)... that it is quite an individual thing... because it is an art rather than a science, the extraction of the pertinent details from the real world situation... it is very much our own interpretations that what the pertinent details are...

A16: The primary problem I always encountered as a member of the team is a lack of clear vision of what you supposed to do...

A16: So as a team member the primary problem always was what to do, when to do, and what is the highest priority... and everybody has his own opinion that what is the most important... so if you have a clear set of objectives then that helps relationship with other team members...

A15: you can view model development solely as an exercise of communication... everyone got his own idea of how system works inside his head... so constructing a model outside everyone's head so every can see the same thing and play with it and manipulate it in different ways... communication is the most expensive part of whole modelling activity... not only with the modellers but you got to communicate with domain experts, users and other people... you have to communicate the whole time...
\end{abstract}

\title{
5.2 Documentation practice
}

The amount and type of documentation produced by our participants varies with their context; however, comments in comment boxes or model code seem to be the most important form of documentation for a simulation model, since every participant emphasized on comments. In addition to comments, simulation objectives, model scope, inputs, outputs, influence diagrams, model structure/design, explanation of model working and scenarios for experiments should be documented. However, the extent of documentation produced for a simulation model depends on a number of factors.

Type of simulation project: Most researchers do not document their models and believe their research papers, conference presentations and dissertation serve as model documentation, as A7 says, "I think (my) model is not going to be something that is safety or business critical. At least for me writing of the paper is model documentation". However, if the model has to be produced for a client or in collaboration with the industry, then there can be some formal documentation depending on the requirements by the client and the modellers own aptitude.

Size and length of simulation modelling project: The size and length of a simulation project are also very important factors in determining the extent of documentation produced for a simulation model. Our participants do not produce documentation for small, quick and throw away models. However, documentation may prove to be helpful for medium/large projects depending on the life of the simulation model.

Time and budget: Time and budget allocated for a simulation project may also be a driving force for the amount and extent of documentation produced. Researchers' academic commitments do not allow them enough time for documentation, and for consultants, a 
budget is the primary factor. As A10 puts it, "people don't want to pay for documentation, documentation doesn't get done."

Model users: If the model is to be handed over to the client for long term use and some modifications may be needed in the future, the provision of documentation becomes important. However, in most cases, a simulation modeller is also the user of the simulation model; where he/she provides the results and analysis of a simulation study to the client in the form of reports or presentations which serve as documentation of the model.

Relationship with the client: Some of the participants think that if you have a very close relationship with the client, you may not need to produce a lot of documentation. For example, A8 discusses a model on which he has worked on for 6 years in very close contact with the client, "I was well known and knew the people I was working with... so it was more informal... so I would say there isn't lot of formal documentation specifications... just a user manual..."

\subsection{Maintenance practice}

As most of the models developed by our participants are used for short/medium term, they rarely needed maintenance. However, on occasions, if they have to modify a model developed by someone else, maintainability may be an issue, especially for large models; A8, A12, A13, A14, A15 and A16 say that they have faced problems while maintaining models developed by someone else. They think that poor documentation makes a model very difficult to maintain.

The participants think that comments are the best support in enhancing the maintainability of a simulation model. Some also think that coherent documentation greatly helps maintainability. A5 and A11 say that model structure should be made as modular as possible in order to enhance model's understandability. A6 also suggests that simulation software can support model maintainability by providing a provision for the modular structure of a model. A8 and A18 suggest that separating the data from the model structure can be potentially very helpful to build maintainable models. A15 says that clarity and simplicity and are also very important aspects of a model's maintainability.

\subsection{Evaluation of models}

Most participants consider validation and verification of models as being evaluation. Moreover, for some participants, such as A13, client satisfaction is the prime parameter for evaluating a model as shown in the Exhibit 4. Two participants, A9, A10 and A11 also say that usability and performance are an implicit part of their model evaluation; however, no formal approach is followed to evaluate this. As suggested by A4 and A18, a model's documentation, usefulness, usability and maintainability are some of the measure of evaluation that should be taken into account.

Some consultants, as shown in the Exhibit 4, believe that clients are more interested in the results or outcome of a simulation model than the actual process of development and evaluation of the model. A12 believes that commercial world does not really care about evaluation unless it is associated with financial loss or gain.

Most of the researchers believe the ultimate aim of their models is to get a publication out of it. Their models are presented and published in conferences and journals through a peer review process; that help them evaluating their models. They say that dissertation defence also serves as a form of evaluation for their models. Although documentation, maintainability, usability and performance are interesting aspects of a model evaluation, their academic and administrative responsibilities do not allow them much time to spend on these aspects of model evaluation (apart from validation and verification) unless it has some research value. 
Exhibit 4: Quotes from transcripts about simulation model evaluation.

\footnotetext{
A12: "Well, I think it (not doing formal evaluation) is certainly an intellectual compromise...I think on the other hand you can know by looking at the commercial benefits that you are producing...so things round about between the two really..."

A13: "evaluation from my point if view would be customer satisfaction... you know it doesn't really matter whether a model is $60 \%$ or $100 \%$ accurate if the customer is happy with your analysis and results"

A10: Surely we monitor performance for the models because that's important and in terms of usability, we are quite sensitive about that

A10: Yes, we ask the user, do you like it? He may say yes or no... so we can ask what you don't like and they tell us and we change (laughing)...
}

\section{DISCUSSION OF RESULTS}

These results provide a general picture of a range of contextual factors and how they can affect simulation practice. Knowledge of the possible effect of context on simulation practice can be particularly helpful for novices to adapt their simulation practice according to the varying contexts.

The results suggest, since the majority of the models developed by our participants are small/medium and not very complex, that most models are developed by individual modellers. However, in larger projects, responsibilities may be divided into a number of different people in a team which may include a process mapper, data collector, model designer and developer. This supports Robinson's [2] assertion that the majority of the models are developed by a single modeller. Moreover, amongst our participants, the modeller is also usually the model user where he/she runs experiments on a validated and verified model and presents the results to the client. Since, in most cases, they do not hand over the models to the client for long term use, they don't have to worry much about model's maintenance, usability and interface design.

Since the majority of the models developed by our participants are small/medium in size, low/medium in complexity and don't take very long to develop, they do not formally design the structure of the model before developing it on a computer. This supports the findings of Foss et al. [15] that most modellers do not design their models formally. As A8 says, for small projects, spending too much time on formally designing the simulation model may not be feasible in terms of cost and time; however, formally designing a large model which is to be used for a long period of time may save valuable time both during and after development. As explained by A12, the nature of simulation modelling does not require him to design a model structure prior to constructing the model; because on most occasions, in the initial stage of a project, neither the modeller nor client fully understand the problem that is to be simulated; therefore producing a design for something for which requirements are not clear may not be easy.

Although there are increasing concerns amongst researchers about the growing simulation model complexity and size and its implications [16-18], our results suggest that simulation model size and complexity is an obscure concept for which no generally agreed metrics exist. Therefore, perceptions on model size and complexity may differ depending on one's background, experience and worldview on simulation and modelling. A model being big and complex for one may be very easy for another to understand or vice versa. Although simulation model size and complexity seem to have huge implications on simulation modelling practice [19], we could not find any considerable study in the literature that explores the relationship of simulation model size and complexity with simulation modelling practice. Our findings suggest that the simulation research community needs to pay more attention towards understanding the phenomenon of simulation model complexity and size, and how it may affect simulation practice. 
Although model maintenance is a topic of greater interest and debate in the defence and military simulation community [20], our participants are not too concerned with the maintainability of their models, since their models are usually small/medium and rarely reused. However, maintainability may become important if the models are needed to be evolved and enhanced in future.

Most of our participants do not document their models in a formal way and the extent of documentation varies depending on each individual context. This finding supports the view of Salt [21] that defence modellers are obsessed with reuse and documentation, while civilians do not bother reusing and documenting their models. Documentation has a crucial role if a model is to be reused and maintained in future [6]. Foss et al. [15] believe that most models are rarely reused because they are poorly documented; and poor documentation hinders maintainability of a model. However, Taylor et al. [22] and Robinson et al. [23] believe that it in contrast to defence simulation, it is often more difficult to reuse an old model as opposed to building a new model from scratch; because in the business world, the objectives and requirements of a model may change very rapidly; therefore, a model build against previous objectives and requirements may not be reusable for a new objective. However, the knowledge and experience gained from an old model is always reused in the new model.

Most of our participants believe that evaluation of a model consists of model validation and verification. Other aspects of evaluation such as quality, usability, utility, performance, documentation and maintainability are not usually considered by our participants. Simulation modelling literature also shows that validation and verification is the main focus in model evaluation for the models developed for business and industry [24]. However, other aspects of model evaluation seem to be taken care of in large scale defence simulation [6]. Moreover, as discussed earlier, most models are used by the modellers themselves; a holistic evaluation will be needed only when the budgets allow and models are to handed over to the client for use.

\section{CONCLUSION AND FUTURE DIRECTIONS}

Exploring the contexts and practices of expert simulation helped understand simulation practice in the real world. The results of this study suggest that the modelling practices of modellers is affected by many contextual factors such as problem domain, model aim and questions to be answered, simulation software, team dynamics, client contact, model $/ \mathrm{problem}$ size and complexity and client requirements. Below, we summarise the results:

- The results suggest that most of the models developed by our participants are small/medium in size and low/medium in complexity. A model's life, complexity, size and simulation software selection may affect simulation practices such as documentation, maintenance, the conceptual design phase and evaluation of simulation models.

- Moreover, most of the participants believe that simulation model size and complexity are related, however, they do not seem to have similar concepts and perceived metrics.

- Simulation software also seems to have a relationship with model complexity and size, as well as the time it takes to develop the model.

- Most of the participants typically develop their models alone, however, on rare occasions when they have to work in a team; communication with other people involved in simulation project appears to be the single biggest problem when a simulation modeller has to work in a team.

- Since most of the participants develop small/medium models, they do not produce any formal design of their models.

- The amount and extent of documentation for a simulation model varies depending on the nature of a simulation project, time and budget, size of simulation model, model life and 
model users. Since modellers rarely need to maintain simulation models, model maintenance is not an issue.

- Client satisfaction with the model results is the key criterion for model evaluation, and validation and verification are the key evaluation activities.

This study, like any other qualitative study, is not without limitations. Given that the data has been collected from a small sample size and drawn mostly through personal contacts, the results do not provide a uniform or view of simulation practice in business and industry but identify some trends and pointers on which future studies can be built. In order to further explore, validate and generalise these findings, a large scale survey of simulation modellers' practice would be useful. This would potentially add to the validity of the findings from this research and allow further insights into the contexts and practices of simulation modellers. Although our participants consisted of both system dynamics and discrete even modellers, they did not identify any difference in simulation practice depending on modelling techniques. However, in future studies, it would be interesting to explore how the modelling practices, especially the modelling process of discrete events and continuous simulation modellers differ. In addition, conducting studies in niches (e.g. defence, manufacturing, healthcare, retail, logistics etc.) of simulation modelling will help further understand the state-of-the-art and state-of-practice discipline in a specific area.

A simulation modeller's context also has a relationship with the modelling process of a modeller. It would be interesting to explore how the process of model development is related with a modeller's context. Moreover, further in-depth studies focussing the areas of validation and verification, documentation, model reuse and conceptual modelling with the modellers' context will be a useful addition to simulation methodology research.

\section{REFERENCES}

[1] Pidd, M. (1999). Just modeling through: A rough guide to modeling, Interfaces, Vol. 29, No. 2, 118-132, doi:10.1287/inte.29.2.118

[2] Willemain, T. R. (1994). Insights on modelling from a dozen experts, Operations Research, Vol. 42, No. 2, 213-222, doi:10.1287/opre.42.2.213

[3] Robinson, S. (2002). Modes of simulation practice: approaches to business and military simulation, Simulation Modelling Practice and Theory, Vol. 10, No. 8, 513-523, doi:10.1016/ $\underline{\text { S1569-190X(02)00117-X }}$

[4] Eriksson, D. M. (2003). A framework for the constitution of modelling processes: A proposition, European Journal of Operational Research, Vol. 145, No. 1, 202-215, doi:10.1016/S03772217(01)00390-3

[5] David, A. (2001). Model implementation: A state of the art, European Journal of Operational Research, Vol. 134, No. 3, 459-480, doi:10.1016/S0377-2217(00)00269-1

[6] Gass, S. I. (1987). Managing the modelling process: A personal reflection, European Journal of Operations Research, Vol. 31, No. 1, 1-8, doi:10.1016/0377-2217(87)90129-9

[7] Ayadi, M.; Costa Affonso, R.; Cheutet, V.; Masmoudi, F.; Riviere, A.; Haddar, M. (2013). Conceptual model for management of digital factory simulation information, International Journal of Simulation Modelling, Vol. 12, No. 2, 107-119, doi:10.2507/IJSIMM12(2)4.233

[8] Murphy, S. P.; Perera, T. D. (2001). Key enablers in the development of simulation, Proceedings of the $33^{\text {rd }}$ Winter Simulation Conference, Vol. 2, 1429-1437, doi:10.1109/WSC.2001.977466

[9] Hollocks, B. W. (2001). Discrete-event simulation: an inquiry into user practice, Simulation Practice and Theory, Vol. 8, No. 6-7, 451-471, doi:10.1016/S0928-4869(01)00028-3

[10] Melão, N.; Pidd, M. (2003). Use of business process simulation: A survey of practitioners, Journal of the Operational Research Society, Vol. 54, No. 1, 2-10, doi:10.1057/palgrave. jors. 2601477

[11] Cochran, J. K.; Mackulak, G. T.; Savory, P. A. (1995). Simulation project characteristics in Industrial settings, Interfaces, Vol. 25, No. 4, 104-113, doi:10.1287/inte.25.4.104 
[12] Tako, A. A.; Robinson, S. (2010). Model development in discrete-event simulation and system dynamics: An empirical study of expert modellers, European Journal of Operational Research, Vol. 207, No. 2, 784-794, doi:10.1016/j.ejor.2010.05.011

[13] Willemain, T. R. (1995). Model formulation: What experts think about and when, Operations Research, Vol. 43, No. 6, 91-102, doi:10.1287/opre.43.6.916

[14] Wang, W.; Brooks, R. J. (2007). Improving the understanding of conceptual modelling, Journal of Simulation, Vol. 1, No. 3, 153-158, doi:10.1057/palgrave.jos.4250024

[15] Foss, B. A.; Lohmann, B.; Marquardt, W. (1998). A field study of the industrial modeling process, Journal of Process Control, Vol. 8, No. 5-6, 325-338, doi:10.1016/S09591524(98)00018-3

[16] Page, E. H.; Nicol, D. M.; Balci, O.; Fujimoto, R. M.; Fishwick, P. A.; L'Ecuyer, P.; Smith, R. (1999). Panel: strategic directions in simulation research, Proceedings of $31^{\text {st }}$ Winter Simulation Conference, Vol. 2, 1509-1520, doi:10.1109/WSC.1999.816887

[17] Law, D. R. (1998). Scalable means more than more: a unifying definition of simulation scalability, Proceedings of the $30^{\text {th }}$ Winter Simulation Conference, Vol. 1, 781-788, doi:10.1109/ WSC.1998.745064

[18] Arthur, J. D.; Sargent, R. G.; Dabney, J. B.; Law , A. M.; Morrison, J. D. (1999). Verification and validation: what impact should project size and complexity have on attendant V\&V activities and supporting infrastructure?, Proceedings of the $31^{\text {st }}$ Winter Simulation Conference, Vol. 1, 148-155, doi:10.1109/WSC.1999.823064

[19] Chwif, L.; Barretto, M. R. P.; Paul, R. J. (2000). On simulation model complexity, Proceedings of the $32^{\text {nd }}$ Winter Simulation Conference, Vol. 1, 449-455, doi:10.1109/WSC.2000.899751

[20] Davis, P. K.; Anderson, R. H. (2004). Improving the composability of Department of defence models and simulations, Journal of Defense Modeling and Simulation, Vol, 1, No. 1, 5-17, doi: $10.1177 / 154851290400100101$

[21] Salt, J. D. (2006). Modes of practice in military simulation, Proceedings of OR 48, 24-35

[22] Taylor, S. J. E.; Lendermann, P.; Paul, R. J.; Reichenthal, S. W.; Strassburger, S.; Turner, S. J. (2004). Panel on future challenges in modeling methodology, Proceedings of the Winter Simulation Conference, Vol. 1, 319-327, doi:10.1109/WSC.2004.1371333

[23] Robinson, S.; Nance, R. E.; Paul, R. J.; Pidd, M.; Taylor, S. J. E. (2004). Simulation model reuse: definitions, benefits and obstacles, Simulation Modelling Practice and Theory, Vol. 12, No. 7-8, 479-494, doi:10.1016/j.simpat.2003.11.006

[24] Shi, P.; Liu, F.; Yang, M. (2010). A modified ANP and its application in simulation credibility evaluation, International Journal of Simulation Modelling, Vol. 9, No. 4, 195-207, doi: $\underline{10.2507 / I J S I M M 09(4) 3.161 ~}$ 